\title{
MedDRA High Level Group Term Code
}

National Cancer Institute

\section{Source}

National Cancer Institute. MedDRA High Level Group Term Code. NCI Thesaurus. Code C117046.

A coded value specifying the high level group term from the Medical Dictionary for Regulatory Activities (MedDRA). 